\title{
Zur Problematik der mammografisch dichten Brust - Positionspapier des AK Mammasonografie der DEGUM*
}

\section{The problem of mammographic breast density - the position of the DEGUM working group on breast ultrasound}

Autoren

Institute
M. P. Mueller-Schimpfle', V. C. Brandenbusch², F. Degenhardt ${ }^{3}$, V. Duda ${ }^{4}$, H. Madjar ${ }^{5}$, A. Mundinger ${ }^{6}$, R. Rathmann ${ }^{7}$, M. Hahn ${ }^{8}$

Die Institutsangaben sind am Ende des Beitrags gelistet.

\author{
Key words \\ - breast \\ - mammography \\ ultrasound \\ - neoplasms \\ - screening
}

\section{Zusammenfassung}

$\nabla$

Die mammografisch dichte Brust ist mit einem erhöhten Risiko einerseits für die Entstehung von Brustkrebs und andererseits für die Maskierung von Karzinomen in der Mammografie korreliert. Dies hat in einer zunehmenden Zahl von Staaten der USA zur gesetzlichen Verpflichtung geführt, die mammografische Brustdichte an die Frau wie auch an den behandelnden Arzt weiterzugeben. Der Arbeitskreis Mammasonografie empfiehlt die Mitteilung der Brustdichte und die Aufklärung der betroffenen Frauen über die Vorteile individualisiert ergänzender Verfahren in Abhängigkeit vom Gesamterkrankungsrisiko der Frau; aufgrund der deutschen Gesundheitsstruktur mit Erfahrung und Qualitätssicherungsprogrammen insbesondere die ergänzende Durchführung einer Mammasonografie unter Sicherung von Struktur-, Prozess-, und Ergebnisqualität. Die Probleme möglicher Überdiagnostik, zusätzlicher Kosten und erhöhten emotionalen Stress sind bei der Aufklärung zu berücksichtigen. Bei familiärer Hochrisikokonstellation sollte eine humangenetische Beratung durchgeführt, eine genetische Testung und intensivierte Früherkennung unter Einbeziehung der MR-Tomografie erwogen werden.

\section{Ausgangssituation \\ $\nabla$}

Die Mammografie stellt die einzige Früherkennungsmethode für Brustkrebs dar, die in unterschiedlichen populationsbezogenen Studien einen positiven Effekt auf die Reduktion der brustkrebsspezifischen Sterblichkeit zeigen konnte [1]. Die Zuordnung der Anteile von mammografischer Früherkennung einerseits und Therapiefortentwicklung andererseits auf die bei Mammakarzinomen beob-

\footnotetext{
*English version online.

\section{Abstract \\ $\nabla$}

Mammographic breast density correlates with breast cancer risk and also with the number of false-negative calls. In the USA these facts lead to the "Breast Density and Mammography Reporting Act" of 2011. In the case of mammographically dense breasts, the Working Group on Breast Ultrasound in Germany recommends explaining the advantages of adjunct imaging to women, depending on the individual breast cancer risk. Due to the particular structure of German healthcare, quality-assured breast ultrasound would be the first choice. Possible overdiagnosis, costs, potentially increased emotional stress should be addressed. In high familial breast cancer risk, genetic counselling and an intensified early detection program should be performed.
Korrespondenzadresse Schimpfle

Klinik für Radiologie,

Neuroradiologie und

Nuklearmedizin, Klinikum

Frankfurt Höchst

Gotenstraße 6-8

65929 Frankfurt am Main

Tel.: ++49/69/31062818

Fax: ++ 49/69/31062511

profmms@web.de achtete Sterblichkeitsreduktion der letzten Jahre ist schwierig zu bestimmen [2]. Dennoch bleibt auch in Zeiten modernster Therapiekonzepte die initiale Tumorgröße bei Diagnosestellung ein wesentlicher und unstrittiger prognostischer Parameter [3]. Somit bleibt folgerichtig die Bedeutung der Bildgebung für die Früherkennung des Mammakarzinoms ungemindert bestehen. Der Effekt kleinerer Tumorgrößen auf mögliche Therapieansätze wird künftig immer differenzierter, nicht zuletzt in Kombination mit molekulargenetischen Tumorund Risikokategorien [4]. Darüber hinaus ergeben sich Hinweise auf eine Steigerung der Effektivität 
der bildgebenden Früherkennung durch größere Individualisierungen in Abhängigkeit vom individuellen Risiko [5].

\section{Brustdichte in der Diagnostik \\ $\nabla$}

Die mammografisch hohe Brustdichte spielt gleich in zweierlei Hinsicht eine wichtige Rolle: erhöhtes Brustkrebsrisiko und Maskierung von Brustkrebs in der Mammografie. Zum einen konnte ein in Metaanalysen 4 -5fach erhöhtes Risiko für die Entstehung von Brustkrebs nachgewiesen werden, wenn Frauen mit sehr niedriger Brustdichte verglichen wurden mit Frauen, die eine sehr hohe Brustdichte aufwiesen [6 - 9]. Zum anderen wirkt sich die Dichte auf die Möglichkeit des Nachweises, vor allem aber der Sicherheit des Ausschlusses eines Mammakarzinoms aus (Sensitivität, negativer Vorhersagewert) [10]. Eine mit der volumetrischen Drüsenkörperdichte abnehmende Sensitivität und zunehmende Zahl falsch positiver Befunde wurde kürzlich auch für die digitale Vollfeldmammografie gezeigt [11].

Aus diesen Gründen entstand in den USA 2011 ein Gesetz (Breast Density and Mammography Reporting Act of 2011), das es den mammografierenden Einrichtungen vorschreibt, sowohl dem zuweisenden Arzt wie auch der Patientin ihre individuelle Brustdichte („patient's individual measure of breast density“) schriftlich mitzuteilen. Dieses Gesetz ist mittlerweile in 21 Staaten der USA verabschiedet und in Kraft. Weitere Staaten arbeiten derzeit an einem Gesetz oder haben es bereits zur Verabschiedung eingebracht [12]. In einer US-amerikanischen Studie an 9232 Frauen in der Nachsorge nach Mammakarzinom, also nicht aus einem populationsbezogenen Screeningkollektiv entstammend, wurde kein Zusammenhang zwischen Brustdichte und Brustkrebssterblichkeit gefunden [13]. Dagegen wurden im Kopparberg-Kollektiv des Schwedischen Screeningprogramms bei 15658 Frauen zwischen 45 und 59 Jahren in einer randomisierten kontrollierten Studie bei Frauen mit dichter Brust sowohl eine erhöhte Brustkrebsinzidenz (relative Risikoerhöhung RR 1,57) als auch eine erhöhte Brustkrebssterblichkeit (RR 1,91) festgestellt [14].

In Deutschland hat sich in den letzten Jahren der BI-RADS-Standard des ACR im Wesentlichen durchgesetzt, wenn auch mit kleinen semantischen oder auch inhaltlichen Modifikationen oder Anmerkungen durch Publikationen von Fachgesellschaften oder Konsensuskonferenzen [15 - 19]. Damit hielt auch die standardisierte Beurteilung der Brustdichte Einzug in Deutschland, wenn diese auch bisher keinen Niederschlag in der Vereinbarung von Qualitätssicherungsmaßnahmen nach $\S 135$ Abs. 2 SGB V zur kurativen Mammografie fand [20]. Hierin ist zwar die Leuchtdichte bei Bildwiedergabegeräten geregelt, zur mammografischen Dichte sucht man vergeblich einen Hinweis.

Dagegen nimmt die S3-Leitlinie bereits seit 2004 Bezug auf die mammografische Dichte im Hinblick auf das Mammakarzinomrisiko wie auch das Risiko der Befundmaskierung [21]. In der 1. Aktualisierung von 2008 wird bereits die ergänzende Mammasonografie nach einer Mammografie mit ACR-Dichtetypen III/IV gefordert [22]. Doch obwohl das Mammografiescreening in Deutschland flächendeckend und mit hohem Qualitätsanspruch gerade auch zur informierten Entscheidung der Frau eingeführt wurde, klafft zur Problematik der unterschiedlichen Brustdichte eine bemerkenswerte Informationslücke. Im offiziellen Downloadbereich des Mammografiescreeningprogrammes finden sich Broschüren und Merkblätter, die keinerlei Hinweise auf das Thema Brustdichte enthalten [23]. Die Entscheidung über die Teilnahme am flächendeckenden, qualitätsgesicherten Mammografiescreening in Deutsch- land soll aber durch die Frau über eine informierte Abwägung von Nutzen und Schaden getroffen werden.

Abzuwarten bleibt, ob sich dies durch eine Entschließung des Deutschen Ärztetages aus dem Jahr 2014 ändern wird. Es heißt in dem Papier: „Die am Mammografie-Screening teilnehmenden programmverantwortlichen Ärztinnen/Ärzte werden aufgefordert, die Mammografie nach dem ärztlichen Standard zu erbringen und den betreuenden Ärztinnen/Ärzten den vollständigen Befund mitzuteilen“. Was mit dem „vollständigen Befund“ gemeint ist, wurde nicht spezifiziert; vermutet werden darf aber, dass hiermit Befundkategorie (nach BI-RADS) und Brustdichte gemeint sind, nicht aber eine detaillierte Befundbeschreibung, die im Rahmen eines populationsbezogenen Screeningprogrammes kaum zu leisten bzw. finanzierbar sein dürfte. Weiterhin wird in der Begründung darauf Bezug genommen, dass die Weiterbetreuung der Patientinnen gewährleistet sein muss. Insbesondere wird auf das Patientenrechtegesetz verwiesen [24].

Es wird in der Entschließung des Ärztetages kein konkreter Bezug zur Brustdichte hergestellt, dieser ergibt sich allerdings zwangsläufig aus dem oben Gesagten. Da die Brustdichte eine wichtige Aussage zum Risiko der Frau für Brustkrebsentstehung wie auch für Brustkrebserkennung darstellt, hat dies potenzielle Folgen für ihre Weiterbetreuung. Hinzu kommt, dass die Brustdichte - nach dem American College of Radiology in 4 Stufen eingeteilt - im Rahmen der Mammografiebefunderstellung standardisiert dokumentiert wird. Dabei zeigt das aktuelle BI-RADS-Lexikon auf, dass die subjektive Dichteabschätzung in 4 Stufen in den USA zu einer über viele Jahre gleichförmigen Verteilung der Dichtedokumentation geführt haben [25].

Eine Sorge im Zusammenhang mit der Weitergabe der Brustdichte an die Klientinnen des deutschen Mammografiescreenings könnte sein, dass in der ungeklärten Evidenz weiterführender Verfahren bei dichter Brust die Frauen mit den Schlussfolgerungen aus ihrer Brustdichte alleine gelassen, ja geradezu in das Netz qualitativ heterogener Erbringer von individuellen Gesundheitsleistungen (IGeL), deren Stellenwert unklar ist, getrieben würden.

Denn zu einer grundsätzlichen Durchführung weiterführender bildgebender Verfahren bei mammografisch dichter Brust asymptomatischer Frauen mit normalem Risiko sind sehr heterogene Meinungen publiziert worden [26-28].

\section{Ergänzende Verfahren bei hoher Brustdichte \\ $\nabla$}

\section{Tomosynthese}

Die Tomosynthese oder 3D-Mammografie konnte in mehreren Studien der letzten Jahre ihre höhere Sensitivität bei gleichbleibender Spezifität gegenüber der Mammografie zeigen, speziell bei Frauen mit erhöhter Brustdichte, allerdings nicht bei Frauen mit extrem dichter Brust, da hier der notwendige Parenchym-/Fettgewebekontrast fehlt [29 - 35]. Die Strahlendosis für die 3D-Mammografie/Tomosynthese liegt je nach Hersteller und Aufnahmeverfahren typischerweise etwas über der einer 2D-Mammografie, bleibt aber innerhalb der Dosisgrenzwerte für die 2D-Mammografie [36].

\section{Ultraschall}

Eine Reihe von Studien konnte belegen, dass der Ultraschall insbesondere in der dichten Brust Karzinome zusätzlich zur Mammografie erkennen kann [37-42]. Abhängig vom Studiendesign und zugrundeliegender Risikoprävalenz wurde die Anzahl zusätzlich durch Ultraschall entdeckter Mammakarzinome mit bis 4,6/1000 untersuchte Frauen angegeben ( $\bullet$ Tab. 1). Die S3-Leitlinie für das 


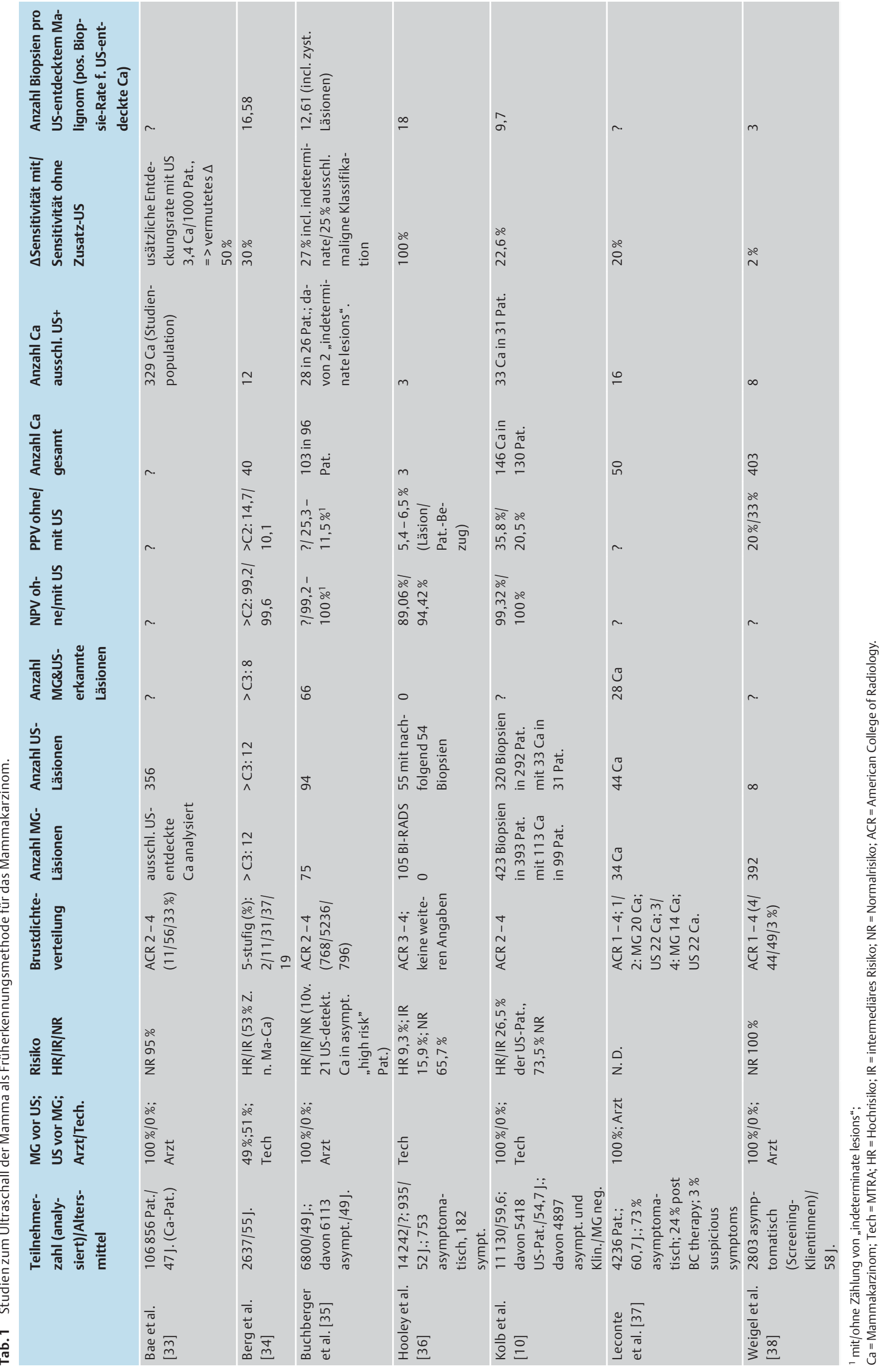


Mammakarzinom fordert daher bereits in der Früherkennungsleitlinie aus 2008 den regelhaften Einsatz der Mammasonografie bei mammografisch dichter Brust (ACR-Dichte 3,4) [22]. Jedoch muss betont werden, dass sich die Forderung nach einer Sonografie bei ACR $3 / 4$ bei einem Evidenzlevel von $3 \mathrm{~b}$ auf eine allgemeine Früherkennung bezieht und eine generelle Übertragung auf das Kollektiv der Klientinnen im Rahmen des Mammografiescreeningprogrammes (Alter 50 - 69 Jahre) nur auf einer ungesicherten Evidenzgrundlage möglich ist.

Auch in der neuesten Leitlinienempfehlung der Arbeitsgemeinschaft Gynäkologische Onkologie wird der Ultraschall als ergänzende Maßnahme bei dichter Brust auf einem Evidenzlevel 2b mit einem Empfehlungsgrad B empfohlen [43]. Im österreichischen Mammografiescreening kommt der Ultraschall als optionale Zusatzbildgebung bei durch den Erstbefunder erkannter Indikation (dichte Brust oder unklarer Befund) unmittelbar nach der Mammografie zur Anwendung [44]. Der Ultraschall ist damit integraler Bestandteil des österreichischen Mammografiescreenings geworden.

Umgekehrt bewertet der IGeL-Monitor „den Ultraschalls der Brust zur Krebsfrüherkennung für Frauen mit unbekannter Brustdichte“ als „unklar“ [45]. Interessanterweise bewerben einzelne Krankenkassen die Mammasonografie zur Früherkennung im Rahmen von Sonderprogrammen demgegenüber doch als erstattungsfähige Leistung [46].

Wichtig ist die Kenntnis der eigentlichen Definition der individuellen Gesundheitsleistung. Zitat aus dem IGeL-Monitor: „IGeL sind alle ärztlichen Leistungen, die per Gesetz nicht zum festgeschriebenen Leistungskatalog der gesetzlichen Krankenversicherung (GKV) gehören. Hierzu zählen Aufgaben, die per se nicht in den GKV-Bereich fallen wie beispielsweise Atteste oder Reiseimpfungen. Zum weitaus größeren Teil sind IGeL jedoch medizinische Maßnahmen zur Vorsorge, Früherkennung und Therapie von Krankheiten, für die eine ganz wesentliche Voraussetzung, die das Gesetz fordert, von offizieller Seite nicht festgestellt wurde, nämlich dass sie dem anerkannten medizinischen Standard entsprechen." [47].

Da der durch die additive Mammasonografie erhöhten Krebsdetektionsrate eine erhöhte Biopsierate gegenüber steht, bleibt die Frage nach dem persönlichen Nutzen für die individuelle Frau offen und das Kosten-Nutzen-Verhältnis für die Gesellschaft möglicherweise im Rahmen der gesetzlichen Krankenversicherung kritisch zu bewerten. In einer risikoadaptierten oder individualisierten Früherkennungssituation jedoch sind die eindeutig erhöhte Sensitivität des Ultraschalls, die im Vergleich zu den vorgenannten Verfahren wesentlich bessere Verfügbarkeit in Kombination mit den in Deutschland bestehenden Qualitätssicherungsmaßnahmen (KBV, DEGUM) deutliche Argumente für den Ultraschall (s. hierzu auch weitere Ausführungen in $[48,49]$ ).

Auch die in Deutschland ohne Zeitverzögerung durchführbare interventionelle Abklärung eines unklaren sonografischen Befundes ermöglicht, anders als bei der stereotaktischen, tomosynthese- oder gar MRT-gesteuerten Abklärung, eine deutlich verkürzte Wartezeit gegenüber den Alternativmethoden. Gerade die Wartezeit und die psychische Belastung in der Wartezeit stellt aber eines der Hauptargumente im Sinne möglicher Schäden für die Frau durch Überdiagnostik dar.

\section{MRT = Kernspintomografie}

Die MRT der Mammae, die in der Mitte der 80er-Jahre des 20. Jahrhunderts in Deutschland durch W. A. Kaiser und S. H. Heywang entwickelt wurde, konnte in den Folgejahren ihre gegenüber den anderen Verfahren höhere Sensitivität unter Beweis stellen [50]. Allerdings können aufgrund möglichen Rückgangs der Spezifität auch eine erhöhte Anzahl nicht maligner Befunde als abklärungswürdig eingestuft werden, was zu zusätzlichen Maßnahmen und unnötigen Biopsien führen kann. Die typischen MRT-Kosten liegen derzeit etwa 10fach über den typischen Sonografiekosten und 5 fach über den typischen Mammografiekosten. International wie auch in Deutschland wird die MRT der Mammae zur Früherkennung für Frauen mit sehr hohem Risiko empfohlen $(>20$ 30 \% Lebenszeitrisiko für Brustkrebs) [51 - 53]. Mehrere Experten empfehlen die MRT auch für Frauen mit intermediärem Risiko (15-30\%) und dichter Brust $[18,54]$.

\section{Empfehlung für Frauen mit hoher Brustdichte \\ $\nabla$}

Eindeutig sind die Empfehlungen in der Abklärungssituation. Hier spielt der Ultraschall unbestritten die zentrale Rolle für Diagnosestellung und bildgesteuerte Abklärung mittels Nadelbiopsie. Dies zeigt sich beispielsweise in den eindeutigen Zahlenverhältnissen sonografisch versus stereotaktisch gesteuerter Biopsien selbst im Mammografiescreening. Im Evaluationsbericht des Jahres 2010 werden für die Erstuntersuchungen 10691 ultraschallgesteuerte Biopsien und 6725 Biopsien unter Röntgenkontrolle angegeben, wohlgemerkt nach primär mammografischer Auffälligkeit [55]. Für die wiedereinbestellten Frauen (PPV I) lag die Karzinomrate bei 13,9\%; für die Frauen, die nach weiterer Abklärung incl. Ultraschall weiterhin eine Biopsieindikation aufwiesen, stieg der Karzinomanteil (PPV II) um das 3,6fache auf 50,4\%. Daraus folgert, dass der Ultraschall in der korrekten Indikationsstellung und qualitätsgesicherten Anwendung offensichtlich in der Lage ist, den PPV zu steigern und nicht etwa, wie einzelne Autoren glauben machen wollen, der Ultraschall den PPV sui generis senkt. Ähnliche Erfahrungen scheint man nach Einführung der verpflichtenden Dichtemitteilung und konsekutiv ergänzend eingesetztem Ultraschall in den USA zu machen, wo von einer Lernkurve und rückläufigen falsch positiven Befunden berichtet [56, 57].

Und was kann den Frauen mit dichter Brust (ACR-Dichte 3/4 bzw. C/D) in der Früherkennungssituation (Individualvorsorge oder Screening) nun empfohlen werden? Die große bundesweite Erfahrung mit der Sonografie, die gute Verfügbarkeit, die vorhandenen Kriterien einer Qualitätssicherung, die belegten Mammakarzinomdetektionsraten durch Ultraschall, die Möglichkeit der zeitnahen Klärung eines verdächtigen Befundes, die geringe physische Belastung der Frau ohne ionisierende Strahlung und ohne Kontrastmittel und nicht zuletzt auch die fortschreitende Entwicklung der sonografischen „Advanced“-Techniken wie auch die immer bessere Hochfrequenz-B-Bild-Darstellung: all dies summiert sich und führt dazu, den Ultraschall den ratsuchenden Frauen mit durchschnittlicher Risikokonstellation präferenziell zu nennen, wenn sie bei dichter Brust eine zusätzliche Erhöhung des negativen Vorhersagewertes wünschen. Je höher die Prä-Testwahrscheinlichkeit (sprich: das individuelle Erkrankungsrisiko zum Zeitpunkt der Vorstellung) ansteigt, desto eher wird sich - in Abwägung der medizinischen Konstellation wie auch der unterschiedlichen Aspekte deutscher Versorgungsrealität - die MRT als erste Wahl der Früherkennungsstrategie positionieren.

Dass diese Empfehlung an die Erfüllung qualitativer Untersucherstandards gebunden ist, versteht sich von selbst, und sollte den ratsuchenden Frauen bei der Suche nach qualitätsgesicherten Einrichtungen unbedingt mit auf den Weg gegeben werden (KBV-Vereinbarung/DEGUM-/DRG-Qualifikationen). 


\section{Institute}

1 Radiology, Neuroradiology and Nuclear Medicine, Frankfurt Höchst Hospital, Frankfurt am Main

Radiology, Diagnostic Breast Center Turmcarree, Frankfurt am Main

Gynecology, Franziskus Hospital, Bielefeld

4 Gynecology, University of Marburg

Gynecology, DKD, Wiesbaden

6 Breast Center, Marienhospital, Osnabrück

Radiology, Schwarzer Baer Practice, Hannover

Gynecology, University of Tübingen

\section{Literatur}

1 Broeders M, Moss S, Nystroem L. EUROSCREEN Working Group. et al. The impact of mammographic screening on breast cancer mortality in Europe: a review of observational studies. J Med Screen 2012; 19: 14-25

2 Glasziou P, Houssami N. The evidence base for breast cancer screening. Prev Med 2011; 53: 100-102

3 Jones $\mathrm{T}$, Neboori $\mathrm{H}, \mathrm{Wu} \mathrm{H}$ et al. Are breast cancer subtypes prognostic for nodal involvement and associated with clinicopathologic features at presentation in early-stage breast cancer? Ann Surg Oncol 2013; 20: $2866-2872$

4 Goncalves $R$, Bose R. Using multigene tests to select treatment for earlystage breast cancer. J Natl Compr Canc Netw 2013; 11: 174-182

5 Onega T, Beaber EF, Sprague BL et al. Breast cancer screening in an era of personalized regimens: a conceptual model and National Cancer Institute initiative for risk-based and preference-based approaches at a population level. Cancer 2014; 120: 2955-2964

6 Sickles EA. The use of breast imaging to screen women at high risk for cancer. Radiol Clin North Am 2010; 48: 859-878

7 Boyd NF, Byng JW, Jong RA et al. Quantitative classification of mammographic densities and breast cancer risk: results from the Canadian National Breast Screening Study. J Natl Cancer Inst 1995; 87: 670-675

8 Byrne C, Schairer C, Wolfe J et al. Mammographic features and breast cancer risk: effects with time, age, and menopause status. J Natl Cancer Inst 1995; 87: 1622 - 1629

9 McCormack VA, dos Santos Silva I. Breast density and parenchymal patterns as markers of breast cancer risk: a meta-analysis. Cancer Epidemiol Biomarkers Prev 2006; 15: 1159-1169

10 Kolb T, Lichy J, Newhouse JH. Comparison of the Performance of Screening Mammography, Physical Examination, and Breast US and Evaluation of Factors that Influence Them: An Analysis of 27,825 Patient Evaluations. Radiology 2002; 225: 165 - 175

11 Wanders JOP, Holland K, Veldhuis WB et al. Effect of volumetric mammographic density on performance of a breast cancer screening program using full-field digital mammography. Insights Imaging 2015; 6: (SS 1802), B-1028, S385

12 http://www.areyoudenseadvocacy.org/dense/ Stand 3.2.2015

13 Gierach GL, Ichikawa L, Kerlikowske $K$ et al. Relationship between mammographic density and breast cancer death in the Breast Cancer Surveillance Consortium. J Natl Cancer Inst 2012; 104: 1218-1227

14 Chiu SY, Duffy S, Yen AM et al. Effect of baseline breast density on breast cancer incidence, stage, mortality, and screening parameters: 25-year follow-up of a Swedish mammographic screening. Cancer Epidemiol Biomarkers Prev 2010; 19: 1219-1228

15 Madjar H, Ohlinger R, Mundinger A et al. BI-RADS analoge DEGUM Kriterien von Ultraschallbefunden der Brust - Konsensus des Arbeitskreises Mammasonographie der DEGUM. Ultraschall in Med 2006: 27, $374-379$

16 Müller-Schimpfle M. für die AG Mammadiagnostik der DRG Konsensustreffen der Kursleiter in der Mammadiagnostik am 5.5.2007 in Frankfurt am Main - Thema: Mikrokalk. Fortschr Röntgenstr 2008; 180 : 66-68

17 Müller-Schimpfle MP, Heindel W, Kettritz U et al. Konsensustreffen der Kursleiter in der Mammadiagnostik am 9.5.2009 in Frankfurt am Main - Thema: Herdbefunde. Fortschr Röntgenstr 2010; 182: 671 - 675

18 Müller-Schimpfle MP, Heindel W, Kettritz U et al. Konsensustreffen der Kursleiter in der Mammadiagnostik am 7.5.2011 in Frankfurt am Main - Magnet-Resonanz-Tomografie der Mamma. Fortschr Röntgenstr 2012; 184: $919-924$

19 Müller-Schimpfle M, Heindel W, Kettritz U et al. AG Mamma - Konsensustreffen der Kursleiter in der Mammadiagnostik in Frankfurt am
Main - Standards in Technik und Befundung. Senologie - Zeitschrift für Mammadiagnostik und -therapie 2014; 11: 77 - 82

20 Kassenärztliche Bundesvereinigung. Neufassung der MammographieVereinbarung nach $\S 135$ Abs. 2 SGB V - Vereinbarung von Qualitätssicherungsmaßnahmen nach § 135 Abs. 2 SGB V zur kurativen Mammographie (Mammographie-Vereinbarung). Dtsch Arztebl 2011; 108: A-791/B-643/C-643

21 Schulz KD, Albert US. (Herausgeber) Stufe-3-Leitlinie Brustkrebs-Früherkennung in Deutschland. Germering/München: W. Zuckschwerdt Verlag GmbH, Industriestraße 1, D-82110; 2003, ISBN 3-88603-812-2

22 Albert US. (Herausgeber) Stufe-3-Leitlinie Brustkrebs-Früherkennung in Deutschland. 1. Aktualisierung 2008. Germering/München: W. Zuckschwerdt Verlag GmbH, Industriestraße 1, D-82110ISBN 978-3-88603931-9

23 http://www.mammo-programm.de/downloads/; zuletzt aufgerufen am 3.2.2015

24 Deutscher Ärztetag - Entschließungen zum Tagesordnungspunkt II: Prävention. Dtsch Arztebl 2014; 111: A-1088/B-932/C-882

25 Sickles EA, D’Orsi CJ, Bassett LW et al. ACR BI-RADS ${ }^{\circledR}$ Mammography. In: ACR BI-RADS ${ }^{\circledR}$ Atlas, Breast Imaging Reporting and Data System. Reston: VA, American College of Radiology; 2013

26 ACOG Committee Opinion no. 593: Management of women with dense breasts diagnosed by mammography. Committee on Gynecologic Practice. Obstet Gynecol 2014; 123: 910-911

27 Mauger B. Special Report: Screening Asymptomatic Women with Dense Breasts and Normal Mammograms for Breast Cancer. TEC Assessment Program Volume 28 No. 15, April 2014

28 Hackelöer BJ, Hille $H$. Multimodale Brustkrebsfrüherkennung versus isoliertes Mammografiescreening. Frauenarzt 2014: 55; 948 - 956

29 Skaane P, Bandos AI, Gullien R et al. Comparison of digital mammography alone and digital mammography plus tomosynthesis in a population-based screening program. Radiology 2013; 267: 47-56

30 Ciatto S, Houssami N, Bernardi D et al. Integration of 3D digital mammography with tomosynthesis for population breast-cancer screening (STORM): a prospective comparison study. Lancet Oncol 2013: 14; $583-589$

31 Rose SL, Tidwell AL, Bujnoch LJ et al. Implementation of breast tomosynthesis in a routine screening practice: an observational study. Am J Roentgenol 2013; 200: 1401 - 1408

32 Haas BM, Kalra V, Geisel J et al. Comparison of tomosynthesis plus digital mammography and digital mammography alone for breast cancer screening. Radiology 2013; 269: $694-700$

33 Conant $E$, Wan $F$, Thomas $M$ et al. Implementing digital breast tomosynthesis (DBT) in a screening population: PPV1 as a measure of outcome. Abstract presented at the Radiological Society of North America Scientific Assembly and Annual Meeting, Chicago, IL, USA, December 1-6, 2013. Available from: http://www2.rsna.org/timssnet/rsna/media/pr2013/Conant/abstract/Conant-Tomosynthesis-Abstract-LH.pdf Accessed October 16, 2014

34 Greenberg JS, Javitt MC, Katzen J et al. Clinical performance metrics of 3D digital breast tomosynthesis compared with 2D digital mammography for breast cancer screening in community practice. Am J Roentgenol 2014; 203: 687-693

35 Friedewald SM, Rafferty EA, Rose SL et al. Breast cancer screening using tomosynthesis in combination with digital mammography. JAMA 2014; 311: 2499-2507

36 van Engen R, Bosmans H, Bouwman R et al. Protocol for the Quality Control of the Physical and Technical Aspects of Digital Breast Tomosynthesis Systems. Draft version 0.15. European Reference Organisation for Quality Assured Breast Screening and Diagnostic Services; January 2014. http://www.euref.org/downloads?download=47: euref-tomo-protocol-version-015\&ei=zagmVZHpN8m8aYPwgIgJ\&usg= AFQjCNHH1qbZwswJgPXLQq72B7Fp-Xfrsg; zuletzt aufgerufen am 9.4.2015

37 Bae MS, Moon WK, Chang JM et al. Breast Cancer Detected with Screening US: Reasons for Nondetection at Mammography. Radiology 2014; 270: $369-377$

38 Berg WA, Blume JD, Cormack JB et al. Combined Screening With Ultrasound and Mammography vs Mammography Alone in Women at Elevated Risk of Breast Cancer. JAMA 2008; 299: 2151 - 2163

39 Buchberger W, DeKoekkoek-Doll P, Springer P et al. Incidental findings on sonography of the breast: clinical significance and diagnostic workup. Am J Roentgenol 1999; 173: 921 - 927 
40 Hooley RJ, Greenberg KL, Stackhouse RM et al. Screening US in Patients with Mammographically Dense Breasts: Initial Experience with Connecticut Public Act 09-41. Radiology 2012; 265: 59-69

41 Leconte I, Feger C, Galant C et al. Mammography and subsequent whole-breast sonography of nonpalpable breast cancers: the importance of radiologic breast density. Am J Roentgenol 2003; 180: 1675-1679

42 Weigel S, Biesheuvel C, Berkemeyer S et al. Digital mammography screening: how many breast cancers are additionally detected by bilateral ultrasound examination during assessment? Eur Radiol 2013; 23: 684-691

43 Schreer I, Albert US. Früherkennung und Diagnostik. In: AGO Breast Committee. Diagnosis and Treatment of Patients with Primary and Metastatic Breast Cancer. Recommendations 2015.www.ago-online. de; zuletzt aufgerufen am 09.04.2015

44 http://www.frueh-erkennen.at/Ablauf.html; zuletzt aufgerufen am 9.4.2015

45 http://www.igel-monitor.de/IGeL_A_Z.php?action=abstract\&id=78, erstellt am 20.3.2013; zuletzt aufgerufen am 8.2.2015

46 http://www.krankenkasseninfo.de/boxentest/vorsorge/krebsvorsorge-ueber-den-gesetzlichen-rahmen-hinaus-27; zuletzt aufgerufen am 8.2.2015

47 www.igel-monitor.de/09_FAQ_Liste_final.pdf; zuletzt aufgerufen am 8.2.2015

48 Hackelöer BJ, Hille $H$. Multimodale Brustkrebsfrüherkennung versus isoliertes Mammografiescreening. Frauenarzt 2014; 55: 948-956

49 Würstlein R, Degenhardt F, Duda V et al. Evaluation of the Nationwide DEGUM Breast Ultrasound Training Program. Ultraschall in Med 2014; 35: $345-349$
50 Lee CH, Dershaw D, Kopans D et al. Breast Cancer Screening With Imaging: Recommendations From the Society of Breast Imaging and the ACR on the Use of Mammography, Breast MRI, Breast Ultrasound, and Other Technologies for the Detection of Clinically Occult Breast Cancer. J Am Coll Radiol 2010; 7: 18-27

51 Houssami N, Lord SL, Ciatto S. Breast cancer screening: emerging role of new imaging techniques as adjuncts to mammography. MJA 2009; 190: $493-498$

52 Lee JM, McMahon PM, Kong CY et al. Cost-effectiveness of Breast MR Imaging and Screen-Film Mammography for Screening BRCA1 Gene Mutation Carriers. Radiology 2010; 254: 793 -800

53 Schmutzler R, Schmidt M. Brustkrebsrisiko und Prävention. In: AGO Breast Committee. Diagnosis and Treatment of Patients with Primary and Metastatic Breast Cancer. Recommendations 2015.www.ago-online.de; zuletzt aufgerufen am 09.04.2015

54 American Cancer Society recommendations for early breast cancer detection in women without breast symptoms. http://www.cancer.org/ cancer/breastcancer/moreinformation/breastcancerearlydetection/ breast-cancer-early-detection-acs-recs. Last Medical Review: 09/10/ 2014. Last Revised: 9/10/2014. Zuletzt aufgerufen am 10.2.201

55 Malek D, Kääb-Sanyal V, Wegener B. Evaluationsbericht 2010 - Ergebnisse des Mammographie-Screening-Programms in Deutschland. Berlin: Kooperationsgemeinschaft Mammographie; 2014

56 Destounis S, Arieno A, Morgan R. Initial experience with the new york state breast density inform law at a community-based breast center. J Ultrasound Med 2015; 34: $993-1000$

57 Weigert J, Steenbergen S. The connecticut experiments second year: ultrasound in the screening of women with dense breasts. Breast J 2015; 21: $175-180$ 